\title{
The association of endogenous hormone concentrations and bone mineral density measures in pre- and perimenopausal women of four ethnic groups: SWAN*
}

\author{
M.R. Sowers $\cdot$ J.S. Finkelstein $\cdot$ B. Ettinger \\ I. Bondarenko $\cdot$ R.M. Neer $\cdot$ J.A. Cauley \\ S. Sherman · G.A. Greendale \\ Received: 10 September 2001 / Accepted: 8 July 2002 \\ (C) International Osteoporosis Foundation and National Osteoporosis Foundation 2003
}

\begin{abstract}
We evaluated bone mineral density (BMD), hormone concentrations and menstrual cycle status to test the hypothesis that greater variations in reproductive hormones and menstrual bleeding patterns in mid-aged women might engender an environment permissive for less bone. We studied 2336 women, aged 4252 years, from the Study of Women's Health Across the Nation (SWAN) who self-identified as African-American $(28.2 \%)$, Caucasian $(49.9 \%)$, Japanese $(10.5 \%)$ or Chinese $(11.4 \%)$. Outcome measures were lumbar spine, femoral neck and total hip BMD by dual-energy X-ray densitometry (DXA). Explanatory variables were estradiol, testosterone, sex hormone binding globulin (SHBG) and follicle stimulating hormone (FSH) from serum collected in the early follicular phase of the menstrual cycle or menstrual status [premenopausal (menses in the 3 months prior to study entry without change in regularity) or early perimenopause (menstrual bleeding in the 3 months prior to study entry but some
\end{abstract}

M.R. Sowers $(\square) \cdot$ I. Bondarenko

Rm 3073, SPH-I Department of Epidemiology,

School of Public Health, University of Michigan,

109 Observatory Street, Ann Arbor, MI 48109, USA

J.S. Finkelstein · R.M. Neer

Endocrine Unit, Department of Medicine,

Massachusetts General Hospital, Boston, MA

B. Ettinger

Division of Research,

Kaiser Permanente Medical Care Program, Oakland, CA

J.A. Cauley

Department of Epidemiology, Graduate School of Public Health, University of Pittsburgh, Pittsburgh, PA

S. Sherman

National Institute on Aging, Bethesda, MD

G.A. Greendale

Division of Geriatrics, UCLA School of Medicine,

Los Angeles, CA, USA

* see Appendix for details change in the regularity of cycles)]. Total testosterone and estradiol concentrations were indexed to SHBG for the Free Androgen Index (FAI) and the Free Estradiol Index (FEI). Serum logFSH concentrations were inversely correlated with BMD $(r=-0.10$ for lumbar spine $[95 \%$ confidence interval $(\mathrm{CI}):-0.13,-0.06]$ and $r=-0.08$ for femoral neck (95\% CI: $-0.11,-0.05)$. Lumbar spine BMD values were approximately $0.5 \%$ lower for each successive FSH quartile. There were no significant associations of BMD with serum estradiol, total testosterone, FEI or FAI, respectively, after adjusting for covariates. BMD tended to be lower ( $p$ values $=0.009$ to 0.06 , depending upon the skeletal site) in women classified as perimenopausal versus premenopausal, after adjusting for covariates. Serum FSH but not serum estradiol, testosterone or SHBG were significantly associated with BMD in a multi-ethnic population of women classified as pre- versus perimenopausal, supporting the hypothesis that alterations in hormone environment are associated with BMD differences prior to the final menstrual period.

Keywords BMD $\cdot$ Estradiol $\cdot$ FSH $\cdot$ Menopause · Menopause transition

\section{Introduction}

Low bone mineral density (BMD) is a major risk factor for osteoporotic fracture. The prevalence of osteoporosis has been estimated to be $20 \%$ among United States women age 50 years and older in the third National Health and Nutrition Examination Survey (NHANES III) based on a cutpoint of -2.5 standard deviations below the mean of a young adult referent population for BMD [1]. This low BMD may arise from inadequate peak bone mass, a decline in bone mass even prior to the menopause, a greater rate of bone loss, particularly immediately following the menopause, or a combination of these characteristics [2]. 
Studies of bone status, especially bone loss, in premenopausal women are conflicting. Some crosssectional studies have reported no evidence of age-related bone difference among women with normal menstrual cycles [3-5], whereas others have reported some age-related bone loss at the femoral neck bone site [6-9]. Because premenopausal bone loss has been estimated to occur at a rate no greater than $1 \%$ per year [10], establishing rates of change is likely to be sensitive to inadequate statistical power to detect small BMD changes without large sample sizes and longitudinal studies. By contrast, rates of perimenopausal bone loss have been reported to be $1-2 \%$ per year [1113] and rates of postmenopausal bone loss are thought to be around $2 \%$ per year [8-14]. The increased likelihood of anovulatory cycles and the greater variation in menstrual patterns in the perimenopause may reflect a hormonal environment that is permissive for bone loss in women prior to the last menstrual period [2].

The age at which losses from peak bone attainment might occur is also poorly characterized. Earlier studies suggested that onset of trabecular bone loss may occur in the third or fourth decade [15]. Recent cross-sectional data indicate that age-related bone loss in both the femoral neck and lumbar spine may begin as early as late adolescence [16] or as late as age 39 years in the femoral neck and age 49 years in the lumbar spine [17]. To determine whether bone peak bone mass is sustained until the age at which the menopause transition commences is complicated because of the difficulty in defining markers that adequately characterize the onset of the menopausal transition.

An expanded understanding of the relationship between peak bone mass levels, hormone concentrations in the perimenopause and the rate of subsequent bone loss will be crucial to evaluating the effect of the public health interventions aimed at sustaining peak bone mass. The Study of Women's Health Across the Nation (SWAN) addresses the following questions in pre- and perimenopausal women: Is BMD lower in women with greater follicle stimulating hormone (FSH) concentrations, an indicator of approaching menopause? Are serum estradiol, testosterone or sex hormone binding globulin (SHBG) concentrations related to lower BMD levels? Are there differences in BMD among SWAN enrollees defined as premenopausal, as compared with those who are perimenopausal, dichotomized according to menstrual cycle frequency criteria? Responses to these questions will contribute to understanding the role of ovarian hormones in sustaining peak bone mass and defining the initiation of bone loss in the menopause $[18,19]$.

\section{Materials and methods}

\section{Study sample}

This is the baseline report from SWAN, a multi-site, longitudinal cohort study of mid-life being conducted in a community-based sample of 3302 women. SWAN participants were from one of five ethnic/racial groups including African-American $(n=935)$, Caucasian $(n=1550)$, Chinese-American $(n=250)$, Hispanic $(n=$ 286) and Japanese-American $(n=281)$ women. The eligibility criteria for the SWAN cohort enrollment included at least one menstrual period in the 3 months prior to screening, age 42 to 52 years, no current use of hormones that affect the ovaries, and selfidentification of membership of one of the five eligible ethnic groups. Women with hysterectomy were precluded from enrollment and these represented $19.9 \%$ of the women contacted in the communities.

Cohort recruitment and enrollment have been described in detail elsewhere [20]. Participants were enrolled in Boston, Chicago, the Detroit area, Los Angeles, Pittsburgh, Oakland, and Hudson County, NJ. All SWAN sites had Caucasian enrollees. Additionally, the Boston, Chicago, Detroit area, and Pittsburgh sites enrolled African-Americans, and the remaining three sites enrolled Japanese, Chinese and Hispanic women, respectively. The study protocols at the Chicago and Newark sites did not include bone densitometry, leaving information from a maximum of 2413 participants for BMD analyses. Of these 2413 enrollees, there were 2336 with useable BMD measures including 2329 women with usable femoral neck BMD data and 2330 women with usable hip measurements. Women with greater body thickness were not excluded (see Data Analysis).

Written informed consent was obtained from all participants and each site's protocol was conducted with approval from an Institutional Review Board.

\section{Measurements}

The study outcome measures were lumbar spine, femoral neck and total hip BMD, in grams per square centimeter. Measurements were made with the Hologic 2000 (Pittsburgh and Oakland) and 4500A (Boston, Detroit area, and Los Angeles) densitometers (Hologic, Waltham, MA). Each site used Osteodyne positioning devices to facilitate the reproducible measurement of the proximal femur [21]. The study-wide quality control program included daily measurement of an anthropomorphic spine phantom at each site, calibration with a spine phantom circulated to sites biannually to provide cross-site and cross-time calibrations, and a site-level review of all participant scans for specified criteria. Synarc, Inc. (Waltham, MA) reviewed $5 \%$ of all scans as well as those scans with potential problems, based on a priori criteria, defining the ultimate status of these particular scans as acceptable, requiring re-analysis or rejected.

Conventional BMD measurements by dual-energy X-ray absorptiometry DXA are expressed in grams per square centimeter and are more properly referred to as 'areal' BMD than true BMD $\left(\mathrm{g} / \mathrm{cm}^{3}\right)$. Areal BMD measures can introduce a bone size scale artifact in that those with greater bone thickness may appear to have greater BMD $[22,23]$. This potential artifact has been addressed by calculating bone mineral apparent density (BMAD) to help account for the geometry of the bones and is useful for considering measures of bone among women of the various racial/ethnic groups who may have differences in bone size and thickness. BMAD was computed for the lumbar spine [22] and femoral neck [23] on the basis of published algorithms.

For the assessment of endogenous hormone concentrations, blood was drawn in days 2-7 of the follicular phase of the menstrual cycle in $86 \%$ of the BMD population, and after a $12 \mathrm{~h}$ fast in $97.2 \%$ of the subjects. Blood was refrigerated for up to an hour, centrifuged, separated and aliquoted. Serum was sent to the SWAN laboratory at the University of Michigan for determination of estradiol $\left(E_{2}\right)$, testosterone $(T)$, sex hormone binding globulin (SHBG) and follicle stimulating hormone (FSH) concentrations. The regression parameter estimates relating hormone concentrations to BMD were unaffected by the inclusion or exclusion of women with blood drawn outside the $2-7$ day window $(n=327)$; therefore, the data presented includes all women.

Hormone assays were conducted using the ACS-180 automated analyzer (Bayer Diagnostics, 115 Norwood Park South, Norwood, 
MA). Serum estradiol concentrations were measured with a modified, off-line ACS:180 ( $\left.\mathrm{E}_{2}-6\right)$ immunoassay. Inter- and intra-assay coefficients of variation averaged $10.6 \%$ and $6.4 \%$, respectively over the assay range. Serum FSH concentrations were measured with a two-site chemiluminometric immunoassay using constant amounts of two monoclonal antibodies provided by Bayer Diagnostics. Each antibody is directed to different regions on the beta subunit (one coupled to paramagnetic particles and the other labeled with DMAE) with specificity for intact FSH. Inter- and intraassay coefficients of variation were $12.0 \%$ and $6.0 \%$, respectively. Testosterone concentrations were determined with the ACS: 180 total testosterone assay modified to increase precision in the lower ranges. The resulting assay includes competitive binding of a DMAE-labeled testosterone derivative to a rabbit polyclonal antitestosterone antibody premixed with monoclonal anti-rabbit IgG antibody immobilized on the solid-phase paramagnetic particles. Inter- and intra-assay coefficients of variation were $10.5 \%$ and $8.5 \%$, respectively.

The de novo two-site chemiluminescent assay for SHBG involved competitive binding of DMAE-labeled SHBG to a commercially available rabbit anti-SHBG antibody and a solid phase of goat anti-rabbit IgG conjugated to paramagnetic particles. Interand intra-assay coefficients of variation were $9.9 \%$ and $6.1 \%$, respectively.

Total testosterone was indexed to SHBG to calculate the Free Androgen Index $(\mathrm{FAI}=100$ total testosterone $/ 28.84 \times \mathrm{SHBG})$. Likewise, total estradiol was indexed to SHBG to calculate the Free Estradiol Index $(\mathrm{FEI})=100 \times$ total estradiol $/ 272.11 \times$ SHBG.

Menstrual cycle status was classified as either premenopausal (menses in the 3 months prior to study entry without change in regularity) or early perimenopause (menstrual bleeding in the 3 months prior to study entry but some change in the regularity of cycles), based on the self-reported regularity of menstrual bleeding.

Measures of weight and height from a calibrated electronic or balance beam scale and stadiometer were used to calculate body mass index $\left(\mathrm{BMI}, \mathrm{kg} / \mathrm{m}^{2}\right)$. Self-administered questionnaires were used to assess current smoking status (never/past/present). Physical activity (a summary score of active living, home, recreational physical activity, plus work activity) was estimated by modifying a published and validated instrument [24]. The modification involved reducing the number of activities about which extensive information was gathered from five activities to three in order to focus interview time for data collection that was most likely to yield discriminatory information. Using regression approaches, we identified that the following factors did not influence the association of hormones with BMD and, therefore, were not incorporated into the statistical models: self-reported hypercalcemia, anorexia or bulimia, tamoxifen use, depo-provera use, glucocorticoid use, history of hyperthyroidism, thiazide use, uterine procedures including D\&C, ever use (yes/no) of any estrogen or estrogen/progestin pill or patch (including oral contraceptives or hormone therapy), prior amenorrhea of at least 3 months duration without being pregnant or breastfeeding, duration of oral contraceptive hormone use, and breastfeeding (ever breastfed). In preliminary analysis, associations of BMD with smoking status or educational level were no longer significant following adjusting for ethnicity and BMI, and were not considered in analysis as covariates.

\section{Data analysis}

Data management and data analyses was completed using SAS version 6.12 (SAS Institute, Cary, NC). Univariate statistics were calculated for the BMD and hormone measurements, and the distributions were examined for departure from normality. A natural log or square root (for SHBG) transformation was applied to the continuous hormone measures to address skewness or to satisfy the modeling assumptions such as normally distributed residuals. Transformed means were untransformed for presentation and appropriate adjustments made for the back-transformation of the variance estimates.

Regression analyses were used to evaluate the role of body thickness, day of the menstrual cycle and potential covariates in the relationship between BMD and hormone concentrations. Preliminary analysis was undertaken to determine whether the body thickness of the enrollees (expressed as the d0 value) affected the association between hormone concentrations and BMD levels, but regression parameter estimates between BMD measures and hormones were unchanged by including thicker women. Therefore, all enrollees with BMD data were included in analyses. Preliminary analysis was also used to identify significant covariates prior to assessing the relationships between BMD and the hormones using regression methodology. The important covariates that were identified included body size, ethnicity, clinical site and physical activity.

The relationships between BMD and hormones and SHBG were estimated by correlation analyses and multiple variable linear regression analyses. Analyses were re-run including the day of the menstrual cycle blood drawn to determine whether adjustment for day of cycle had an impact on the association of BMD with the hormones. Mean hormone and SHBG concentrations of pre- and perimenopausal women were evaluated with analysis of variance (ANOVA).

The association of BMD with menopausal status defined by the regularity of menstrual cycling was estimated by regressing BMD on an indicator variable for pre- versus early perimenopausal status while including covariates in the model. Model fit, including appropriateness of transformations, was assessed graphically and using residual analyses. Both $p$ values, based on a two-sided test, and $95 \%$ confidence intervals $(\mathrm{CI})$ are shown.

Hormone concentrations were categorized into quartiles of their respective distributions to depict the association with BMD measures graphically.

\section{Results}

Table 1 describes the BMD characteristics as well as the hormone concentrations of the sample. The mean FSH concentration was $24 \mathrm{mIU} / \mathrm{ml}$ while the mean estradiol concentration was $77 \mathrm{pg} / \mathrm{ml}$ in this sample whose mean age was 46.3 years. Table 2 presents the frequency of categorical variables in the sample including ethnicity, menopausal status and parity. Fifty-four percent of women were classified as premenopausal while $46 \%$ were classified as early perimenopausal. Table 3 presents the BMD and BMAD LSmeans as well as the hormone concentration means associated with pre- and early perimenopausal status. It can be seen that the mean serum FSH concentration (unadjusted for covariates) was approximately $30 \%$ greater in perimenopausal women as compared with the mean value for premenopausal women. In comparison with perimenopausal women, FEI was higher in premenopausal women $(p=0.04)$ while serum testosterone concentrations were significantly lower $(p=0.01)$. At the same time, both BMD and BMAD, measured at all sites, were significantly higher in premenopausal women compared with early perimenopausal women.

The unadjusted Pearson correlation coefficients relating the hormone concentrations and measures of BMD status are shown in Table 4. Serum FSH concentrations were significantly and negatively associated with all BMD measures with the negative correlations ranging from -0.08 to -0.10 . Notably, there were no associations between serum estradiol concentrations and any of the BMD measures. Although there were statistically significant associations of SHBG, total testoster- 
Table 1 Characteristics of SWAN participants, including measures of bone mineral density and serum hormone concentrations, from the baseline assessment

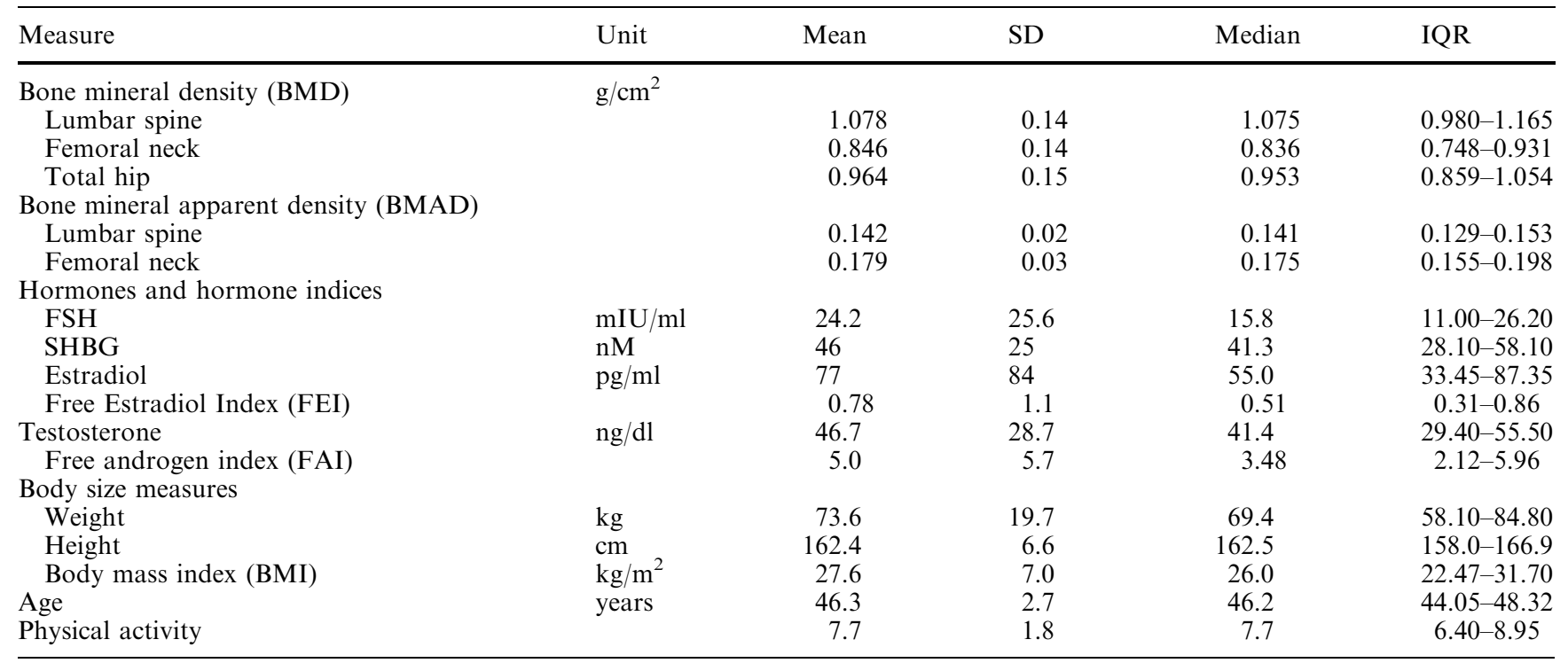

Blood was drawn in days $2-7$ of the follicular phase of the menstrual cycle in $86 \%$ of participants

$\mathrm{IQR}$, interquartile range

Table 2 Frequencies of categorical variables in the SWAN baseline evaluation of pre- and perimenopausal women

\begin{tabular}{|c|c|c|}
\hline Variable & Level & $n(\%)$ \\
\hline Menopausal status & Premenopausal & $1245(54.1)$ \\
\hline \multirow{2}{*}{ Parity } & $1-2$ children & $1182(51.1)$ \\
\hline & More than 2 children & $695(30.1)$ \\
\hline \multirow[t]{2}{*}{ Race/ethnic group } & African-American & $659(28.2)$ \\
\hline & Caucasian & $1166(49.9)$ \\
\hline \multirow[t]{3}{*}{ Smoking status } & Never & $1351(58.7)$ \\
\hline & Past only & $575(25.0)$ \\
\hline & Present & $374(16.3)$ \\
\hline \multirow[t]{3}{*}{ Education } & Less than high school & $94(4.1)$ \\
\hline & High school & $408(17.8)$ \\
\hline & Greater than high school & $782(34.0)$ \\
\hline Anorexia & No & $2309(99.0)$ \\
\hline \multirow[t]{2}{*}{ Bulimia } & Yes & $11(0.5)$ \\
\hline & No & $2323(99.5)$ \\
\hline \multirow[t]{2}{*}{ Ever use of oral contraceptives } & Yes & $1709(73.2)$ \\
\hline & No & $617(26.4)$ \\
\hline \multirow[t]{2}{*}{ Ever use of an estrogen/progestin combination (except oral contraceptives) } & Yes & $15(0.6)$ \\
\hline & No & $2295(98.3)$ \\
\hline \multirow[t]{2}{*}{ Employment status } & Unemployed & $425(18.2)$ \\
\hline & Employed & $1911(81.8)$ \\
\hline
\end{tabular}

one, Free Androgen Index and Free Estradiol Index with BMD, as shown in Table 5, these relationships were largely accounted for by body size and ethnicity.

Table 5 shows the associations between hormone concentrations and BMD from multiple variable regression models, after adjusting for the five variables shown to be important covariates, including ethnicity (African-American, Caucasian, Chinese, Japanese), BMI, clinical site, and physical activity score including the indicator variable for employment. Log-transformed serum FSH concentrations were significantly and consistently negatively associated with BMD values ( $p$ 
Table 3 Mean (with 95\% confidence intervals) hormone, sex hormone binding globulin (SHBG), hormone indices, BMD and BMAD associated with pre- and perimenopausal status (defined by the regularity of menstrual bleeding)

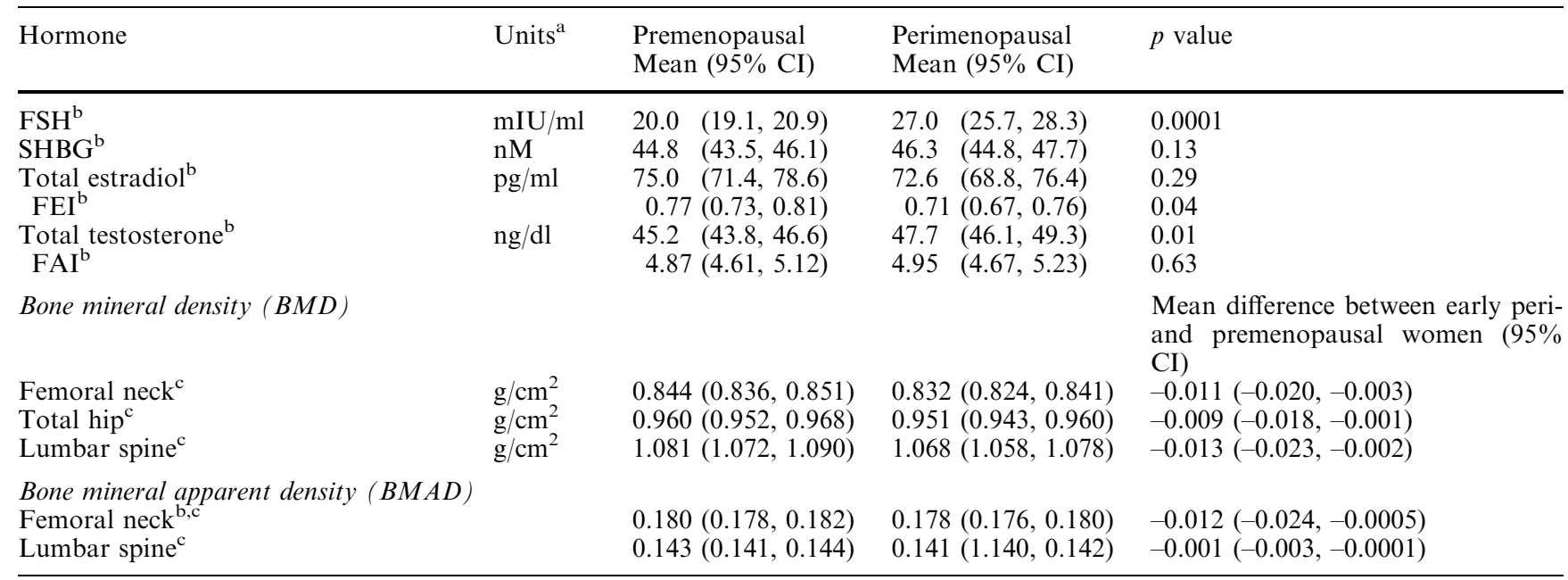

${ }^{a}$ Blood was drawn in days $2-7$ of the follicular phase of the menstrual cycle in $86 \%$ of participants

${ }^{\mathrm{b}}$ The table presents untransformed results; however, all statistical tests were performed with transformed values (square root transformation for SHBG and log transformation for other hormones and femoral neck BMAD)

${ }^{\mathrm{c}}$ Least square means and standard errors, following adjustment for BMI, ethnicity, clinical site, and physical activity with analysis of covariance

Table 4 Pearson correlations (with 95\% confidence intervals) indicating the direction and strength of the unadjusted associations between bone mineral density (BMD) or bone mineral apparent density (BMAD) and serum reproductive hormones ${ }^{\mathrm{a}}$ or their indices

\begin{tabular}{|c|c|c|c|c|}
\hline \multirow{2}{*}{$\begin{array}{l}\text { Bone mineral apparent density } \\
\text { (BMAD) }\end{array}$} & & \multicolumn{3}{|c|}{ Pearson coefficient $(95 \% \mathrm{CI})$} \\
\hline & & Lumbar spine & Femoral neck & \\
\hline $\begin{array}{l}\text { FSH } \\
\text { SHBG } \\
\text { Total estradiol } \\
\text { FEI } \\
\text { Total testosterone } \\
\text { FAI }\end{array}$ & $\begin{array}{l}(\mathrm{mIU} / \mathrm{ml}) \\
(\mathrm{nM}) \\
(\mathrm{pg} / \mathrm{ml}) \\
(\mathrm{ng} / \mathrm{dl})\end{array}$ & $\begin{array}{c}-0.10(-0.13,-0.06) \\
-0.16(-0.19,-0.12) \\
-0.02(-0.05,0.02) \\
0.08(0.05,0.12) \\
0.05(0.02,0.08) \\
0.14(0.10,0.17)\end{array}$ & $\begin{array}{c}-0.08(-0.11,-0.05) \\
-0.13(-0.17,-0.10) \\
-0.01(-0.04,0.03) \\
0.08(0.05,0.12) \\
0.02(-0.02,0.05) \\
0.10(0.07,0.13)\end{array}$ & \\
\hline \multirow{2}{*}{$\begin{array}{l}\text { Bone mineral apparent density } \\
\text { (BMAD) }\end{array}$} & & \multicolumn{3}{|c|}{ Pearson coefficient $(95 \% \mathrm{CI})$} \\
\hline & & Lumbar spine & Femoral neck & \\
\hline $\begin{array}{l}\text { FSH } \\
\text { SHBG } \\
\text { Total estradiol } \\
\text { FEI } \\
\text { Total testosterone } \\
\text { FAI }\end{array}$ & $\begin{array}{l}(\mathrm{mIU} / \mathrm{ml}) \\
(\mathrm{nM}) \\
(\mathrm{pg} / \mathrm{ml}) \\
(\mathrm{ng} / \mathrm{dl})\end{array}$ & $\begin{array}{c}-0.09(-0.13,-0.06) \\
-0.14(-0.17,-0.11) \\
-0.01(-0.04,0.03) \\
0.08(0.05,0.12) \\
0.05(0.01,0.08) \\
0.12(0.09,0.16)\end{array}$ & $\begin{array}{c}-0.10(-0.13,-0.06) \\
-0.15(-0.18,-0.12) \\
-0.01(-0.04,0.03) \\
0.10(0.06,0.13) \\
0.04(0.01,0.07) \\
0.12(0.09,0.16)\end{array}$ & $\begin{array}{c}-0.10(-0.14,-0.07) \\
-0.18(-0.21,-0.15) \\
-0.02(-0.05,0.02) \\
0.10(0.07,0.13) \\
0.07(0.03,0.10) \\
0.16(0.13,0.20)\end{array}$ \\
\hline
\end{tabular}

${ }^{a}$ Blood was drawn in days $2-7$ of the follicular phase of the menstrual cycle in $86 \%$ of participants

values were 0.0002 to 0.0011 , depending upon the BMD site) indicating that higher FSH concentrations were associated with lower BMD, consistent with the crude associations reported in Table 4. As shown in Fig. 1, the multiply adjusted spine BMD values were approximately $0.5 \%$ lower for each increasing quartile of FSH increase. Similar patterns were evident in the total hip and femoral neck values.

SHBG remained negatively associated with BMD following adjustment for BMI, ethnicity, clinical site and physical activity (Table 5), though the associations were statistically significant only with the lumbar spine or lumbar spine BMAD ( $p$ values of 0.01 and 0.016 , respectively). Further, the relation between SHBG and BMD measures were not linear as shown in Fig. 1.

After adjustment for BMI, ethnicity, clinical site and physical activity, there were no significant independent associations of serum estradiol, total testosterone or their SHBG-adjusted indices, FEI and FAI, respectively with the BMD measures (Table 5, Fig. 1). Analysis indicated that the crude associations presented in Table 4 were largely accounted for by ethnicity and body size. 
Table $5 \beta$-coefficients, partial correlation coefficients and $p$ values ${ }^{\mathrm{a}}$ relating BMD to hormone concentrations ${ }^{\mathrm{b}}$ and free androgen and estrogen indices (FAI, FEI) ${ }^{\mathrm{b}}$

\begin{tabular}{|c|c|c|c|c|c|c|c|c|c|}
\hline & \multicolumn{3}{|l|}{ Femoral neck } & \multicolumn{3}{|l|}{ Total hip } & \multicolumn{3}{|l|}{ Lumbar spine } \\
\hline & $\beta$-coefficient & $\begin{array}{l}\text { Partial } \\
\text { correlation }\end{array}$ & $p$ value & $\beta$-coefficient & $\begin{array}{l}\text { Partial } \\
\text { correlation }\end{array}$ & $p$ value & $\beta$-coefficient & $\begin{array}{l}\text { Partial } \\
\text { correlation }\end{array}$ & $p$ value \\
\hline${ }_{\log } \mathrm{FSH}(\mathrm{mIU} / \mathrm{ml})$ & -0.011 & -0.06 & 0.0002 & -0.011 & -0.05 & 0.0009 & -0.012 & -0.06 & 0.001 \\
\hline SHBG (nM) & -0.0001 & -0.02 & 0.16 & -0.0002 & -0.03 & 0.08 & -0.0003 & -0.05 & 0.01 \\
\hline Estradiol $(\mathrm{pg} / \mathrm{ml})$ & -0.0001 & -0.01 & 0.55 & -0.0001 & -0.02 & 0.21 & -0.0001 & -0.03 & 0.07 \\
\hline FEI & 0.0014 & 0.01 & 0.49 & -0.0006 & -0.00 & 0.79 & 0.0007 & 0.00 & 0.77 \\
\hline Testosterone (ng/dl) & -0.0001 & -0.03 & 0.07 & -0.0001 & -0.02 & 0.25 & 0.0000 & 0.00 & 0.96 \\
\hline FAI & 0.0005 & 0.00 & 0.87 & 0.003 & 0.01 & 0.42 & 0.0063 & 0.03 & 0.09 \\
\hline
\end{tabular}

${ }^{\mathrm{a}} p$ values for the independent association of BMD (at all sites) with hormone measures, following adjustment for BMI, ethnicity, clinical site and physical activity

${ }^{\mathrm{b}}$ Blood was drawn in days $2-7$ of the follicular phase of the menstrual cycle in $86 \%$ of participants

BMD measures were somewhat lower ( $p$ values of 0.009 to 0.06 , depending upon the BMD site) in perimenopausal women compared with premenopausal women (see Table 3), following adjustment for covariates. Use of FSH concentrations explained more variability in BMD measures than did the definitions of pre- and perimenopause status based on bleeding regularity.

\section{Discussion}

This study demonstrated that higher serum FSH concentrations were associated with lower measures of BMD in the SWAN multi-ethnic population of pre- and perimenopausal women, supporting the hypothesis that alterations in hormone concentration may promote bone loss before the final menstrual period. These findings persisted whether the BMD was expressed as areal BMD or as BMAD. For each approximate $5 \mathrm{mIU} / 1$ increase in serum FSH, there was approximately a $0.5 \%$ lower BMD value. This relationship was retained after adjustment for race/ethnicity, clinical site, physical activity and body size, variables that were independently associated with BMD. The associations of FSH and BMD measures were observed within each of the four racial/ethnic groups (data not shown). This allowed us to be more confident that the associations with hormone concentrations were unlikely to be due to alternative explanations.

Garton et al. [25] identified that serum FSH in three categories $(<10,10-35$, and $>35 \mathrm{U} / \mathrm{l})$ was inversely

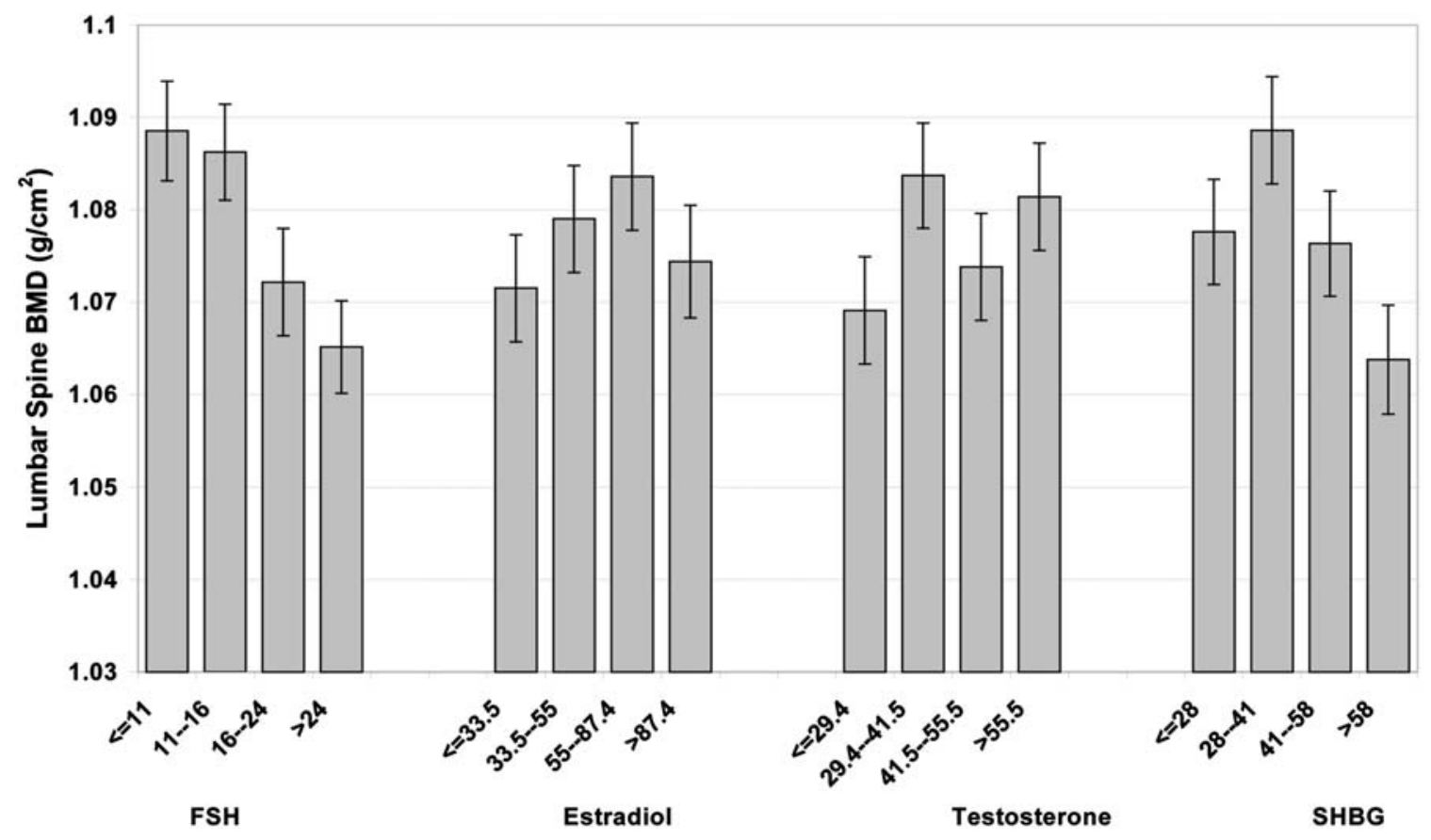

Fig. 1 The LSmeans for lumbar spine BMD values according to quartiles of follicle stimulating hormone (FSH), estradiol, testosterone and sex hormone binding globulin (SHBG) in pre- and perimenopausal women, following adjustment for race/ethnicity, clinical site, BMI and physical activity level 
associated with BMD measured at the spine in the 63 perimenopausal women. Our data suggest that this inverse association with serum FSH and BMD can be observed with FSH concentrations $(<11,11-16,17-24$, $\geqslant 25 \mathrm{mIU} / \mathrm{ml}$ ) in a linear manner. Longitudinal studies will be required to confirm whether these lower concentrations are actually associated with BMD change.

Our data demonstrate that a decline in ovarian function, as indicated indirectly by an increase in serum FSH levels, is associated with lower BMD. Recent data have indicated that inhibin $\mathrm{B}$, a circulating peptide that is produced by granulosa cells in the small ovarian follicles and that functions as a negative regulator of FSH secretion from the pituitary, may be a better marker of ovarian aging than FSH. The early follicular phase serum inhibin B levels were lower in older cycling women than in younger cycling women [26,27], inversely associated with serum FSH levels in perimenopausal women [28-33] and decreased progressively in the years immediately preceding the menopause [30]. Thus, future studies should explore whether inhibin B levels are inversely associated with BMD and, if so, whether the association between serum inhibin B levels and BMD is stronger than the association between FSH levels and $\mathrm{BMD}$. If a rise in FSH concentrations is associated with lower BMD, inhibin B concentrations might be a more sensitive marker of the onset of BMD loss associated with the menopause transition.

Though women classified as being in the early perimenopause (based on the irregularity in menstrual bleeding pattern) had lower BMD than premenopausal women, the use of FSH concentrations explained more variability in BMD levels than did the classification based on menstrual patterns. These current categorical classifications, based on bleeding patterns, may be less sensitive to the physiologic continuum generated by hormone concentrations and their changes through this transitional period. Nonetheless, there were lower BMD levels across the race/ethnic groups when using either the bleeding definition or the hormone concentration models, suggesting an association that occurs apart from those factors that generate significant ethnic differences in BMD [34].

Estradiol deficiency is believed to be responsible for the rapid bone loss following menopause. However, we did not identify an association between total estradiol or free (SHBG-indexed) concentrations and BMD levels in this population of pre- and perimenopausal women. This apparent contradiction can have several explanations. First, we measured estradiol concentrations in the early follicular phase to facilitate interpretation with respect to the stages of the menstrual cycle. However, serum estradiol concentrations in the early follicular phase of the menstrual cycles have less variation than estradiol concentrations in the late follicular phase or luteal phase. This reduced natural variation may obscure the true relationship between circulating estradiol and BMD, particularly as the estradiol concentrations vary greatly between the luteal and follicular phase.
Secondly, this is a cross-sectional examination of estradiol and BMD but the BMD reflects peak bone mass, any BMD change, the lifelong estradiol exposure and any change in estradiol (higher, lower, or highly variable levels) associated with menopause transition. Third, the calculated free estradiol index may inadequately estimate the bioavailable estradiol concentrations, which can also include adrenal sources of estrogen.

Higher serum SHBG concentrations were associated with lower levels of spine BMD, but not with total hip or femoral neck BMD. It has been speculated that higher serum SHBG levels are associated with lower peak bone mass by diminishing the availability of free or biologically active testosterone and estradiol [35]. Although potential associations with estradiol and testosterone were each evaluated for their relationship with BMD measures, neither these hormones nor the indices designed to express the more bioavailable hormone (FEI and FAI) were significantly associated with BMD, particularly after considering the confounding variables of body size and ethnicity.

There are few comparable studies of hormone concentrations and BMD in similarly aged pre- and perimenopausal women. Rannevik et al. [36] observed 160 Swedish women up to 6 years before the menopause and reported no significant associations between hormones and $\mathrm{BMD}$, as characterized by two measures of the distal forearm. A longitudinal study of 75 perimenopausal Midwestern women identified spine and femoral neck BMD loss approximately 2 years before the last menses. Though the authors labeled this bone loss as estrogen-dependent, they did not report whether ovarian and gonadotropin concentrations were associated with BMD change [37].

Comparing our findings on perimenopausal bone loss with those found in other studies is difficult due to lack of consistency in the definition of perimenopausal status. Some studies define perimenopausal status based on a particular age range $[38,39]$, while others defined it base on serum FSH concentrations [6,26]. Findings may also be different depending upon the frequency of hysterectomy in a sample being evaluated. We operationalized ovarian function using both $\mathrm{FSH}$ concentrations and regularity of menstrual cycles. Irrespective of the approach used, we found a modest but consistently lower BMD in women who reported irregular menstrual bleeding as compared to regular menstrual bleeding (defined as premenopausal vs early perimenopausal status) and an even stronger association with FSH.

This is the largest cross-sectional study to date of the relationship between perimenopausal hormone concentrations and BMD, and the first to systematically adjust for concurrent ethnic and body size differences. The SWAN longitudinal study will provide a more detailed description of the hormonal events of the menopausal transition and the ensuing impact on bone mineral change with time. Moreover, the data were 
collected from naturally menstruating women who could be considered representative of their communities [20]; however, this study does not include women who are surgically postmenopausal, and thus their experience is not reflected here. This study extends the current understanding of endogenous hormone concentrations and BMD, as most studies of hormone concentrations and BMD have been conducted in populations of postmenopausal women of European origin. This study does not include an evaluation of the roles of ovulation or progesterone, which in epidemiologic data were related to the level of BMD in healthy premenopausal women [40]. Because these SWAN hormone data are from the early follicular phase of the menstrual cycle, they cannot be effectively used to assess the likely association of progesterone with BMD.

In summary, we conclude that only early follicular FSH hormone concentrations were statistically and consistently associated with lower BMD measured at all sites, after adjusting for the important covariates. This finding suggests that bone loss may be initiated prior to the last menstrual period, a concept that can be tested in the SWAN longitudinal study.

\section{Appendix. SWAN}

The Study of Women's Health Across the Nation (SWAN) was funded by the National Institute on Aging, the National Institute of Nursing Research and the Office of Research on Women's Health of the National Institutes of Health. Supplemental funding from National Institute of Mental Health, the National Institute on Child Health and Human Development, the National Center on Complementary and Alternative Medicine, the Office of Minority Health and the Office of AIDS Research is also gratefully acknowledged.

Clinical Centers: University of Michigan, Ann Arbor, MI (U01 NR04061, MaryFran Sowers, PI); Massachusetts General Hospital, Boston, MA (U01 AG12531, Robert Neer, PI); University of California/Kaiser, Davis, CA (U01 AG12554, Ellen Gold, PI); University of California, Los Angeles, CA (U01 AG2539, Gail Greendale PI); and the University of Pittsburgh, Pittsburgh, PA (U01 AG12546, Karen Matthews, PI).

Laboratory: University of Michigan, Ann Arbor, MI (U01 AG12495, Daniel McConnell, PI) and Medical Research Laboratories (MRL), Highland Heights, KY (subcontract of U01 AG12553, Evan Stein, Director).

Coordinating Center: University of Pittsburgh (Kim Sutton-Tyrrell, PI).

Project Officers: Taylor Harden, Carole Hudgings, Marcia Ory, Sherry Sherman.

Steering Committee Chair: Jennifer L. Kelsey.

The manuscript was reviewed by the Publications and Presentations Committee of SWAN and has its endorsement.

We thank the study staff at each site and all of the women who participated in SWAN.

\section{References}

1. Looker AC, Johnston CC, Wahner HW et al. (1995) Prevalence of low femoral density in older US women from NHANES III. J Bone Miner Res 10: 796-802

2. Sowers MFR, Galuska D (1993) Epidemiology of bone mass in premenopausal women. Epidemiol Rev 15: 374-398

3. Mazess RB, Barden H (1991) Bone density in premenopausal women: effects of age, dietary intake, physical activity, smoking, and birth control pills. Am J Clin Nutr 53: 132-142

4. Lindsay R, Cosman F, Herrington BS et al. (1992) Bone mass and body composition in normal women. J Bone Miner Res 7: $55-63$

5. Hansen MA (1994) Assessment of age and risk factors on bone density and bone turnover in healthy premenopausal women. Osteoporos Int 4: 123-128

6. Sowers MF, Crutchfield M, Bandekar R et al. (1998) Bone mineral density and its change in pre- and perimenopausal white women: the Michigan Bone Health Study. J Bone Miner Res 13: 1134-1140

7. Sowers M, Kshirsagar A, Crutchfield M. Updike S (1991) Body composition, age and femoral bone mass of young adult women. Ann Epidemiol 1: 245-254

8. Ravn P, Hetland ML, Overgaard K et al. (1994) Premenopausal and postmenopausal changes in bone mineral density of the proximal femur measured by dual-energy X-ray absorptiometry. J Bone Miner Res 9: 1975-1980

9. Lofman O, Larsson L, Ross I et al. (1997) Bone mineral density in normal Swedish women. Bone 20: 167-174

10. Baran DT (1994) Magnitude and determinants of premenopausal bone loss. Osteoporos Int Suppl 1: S31-S34

11. Recker RR, Lappe JM, Davies M et al. (1992) Change in bone mass immediately before menopause. J Bone Miner Res 7: 857-862

12. Sowers MFR, Clark M, Hollis B et al. (1992) Radial bone mineral density in pre- and perimenopausal women: a prospective study of rates and risk factors for loss. J Bone Miner Res 7: 647-657

13. Fujiwara S, Fukunaga M, Nakamaura T et al. (1998) Rates of change in spinal bone density among Japanese women. Calcif Tissue Int 63: 202-207

14. Sowers MFR, Clark K, Wallace R et al. (1991) Prospective study of radial bone mineral density in a geographically defined population of postmenopausal Caucasian women. Calcif Tissue Int 48: $232-239$

15. Mazess RB (1982) On aging bone loss. Clin Orthop 165: 239252

16. Matkovic V, Jelic T, Wardlaw GM et al. (1994) Timing of peak bone mass in Caucasian females and its implication for the prevention of osteoporosis: inference from a cross-sectional model. J Clin Invest 93: 799-808

17. Mazess RB, Barden H (1999) Bone density of the spine and femur in adult white females. Calcif Tissue Int 65: 91-99

18. Kulak CAM, Bilezikian JP (1998) Osteoporosis: preventive strategies. Int J Fertil 43: 56-64

19. O’Neill T, Papapoulos S (1997) Can we prevent fractures? Baillieres Clin Rheumatol 11: 565-582

20. Sowers MF, Crawford S, Sternfeld B et al. (2000) Design, survey sampling and recruitment methods of SWAN: a multicenter, multi-ethnic, community-based cohort study of women and the menopausal transition. In: Wren J, Lobo RA, Kelsey J, Marcus R, editors. Menopause: biology and pathobiology. New York: Academic Press

21. Hans D, Duboeuf F, Schott AM et al. (1997) Effects of a new positioner on the precision of hip bone mineral density measurements. J Bone Miner Res 12: 1289-1294

22. Carter DR, Bouxsein ML, Marcus R (1992) New approaches for interpreting projected bone densitometry data. J Bone Miner Res 7: 137-145

23. Katzman DK, Bachrach LK, Carter DR et al. (1991) Clinical and anthropometric correlates of bone mineral acquisition in 
healthy adolescent girls. J Clin Endocrinol Metab 73: 13321339

24. Baecke JA, Burema J, Frijters JE (1982) A short questionnaire for the measurement of habitual physical activity in epidemiological studies. Am J Clin Nutr 36: 936-942

25. Garton M, Martin J, New S et al. (1996) Bone mass and metabolism in women aged 45-55. Clin Endocrinol 44: 563-570

26. Reame NE, Wyman TL, Phillips DJ et al. (1998) Net increase in stimulatory input resulting from a decrease in inhibin B and an increase in activin A may contribute in part to the rise in follicular phase follicle-stimulating hormone of aging cycling women. J Clin Endocrinol Metab 83: 3302-3307

27. Welt CK, McNicholl DJ, Taylor AE et al. (1999) Female reproductive aging is marked by decreased secretion of dimeric inhibin. J Clin Endocrinol Metab 84: 105-111

28. Prior J (1998) Perimenopause: the complex endocrinology of the menopausal transition. Endocr Rev 19: 397-428

29. Burger HG (1999) The endocrinology of menopause. J Steroid Biochem Mol Biol 69: 31-35

30. Seifer DB, Scott RT Jr, Bergh PA et al. (1999) Women with declining ovarian reserve may demonstrate a decrease in day 3 serum inhibin B before a rise in day 3 follicle-stimulating hormone. Fertil Steril 72: 63-65

31. Danforth DR, Arbogast LK, Mrouech J et al. (1998) Dimeric inhibin: a direct marker of ovarian aging. Fertil Steril 70: 119123

32. Muttukrishna S, Child T, Lockwood GM et al. (2000) Serum concentrations of dimeric inhibins, activin A, gonadotrophins and ovarian steroids during the menstrual cycle in older women. Hum Reprod 15: 549-556

33. Burger HG, Cahir N, Robertson DM et al. (1998) Serum inhibins A and B fall differentially as FSH rises in perimenopausal women. Clin Endocrinol 48: 809-813

34. Finkelstein JS, Lee MLT, Sowers MFR et al. (2002) Ethnic variation in bone mineral density in pre- and early perimenopausal women: role of anthropometric and lifestyle factors. J Clin Endocrinol Metab 87: 3057-3067

35. Slemenda C, Longcope C, Peacock M et al. (1996) Sex steroids, bone mass, and bone loss. A prospective study of pre-, peri and postmenopausal women. J Clin Invest 97: 14-21

36. Rannevik G, Jeppsson S, Johnell O et al. (1995) A longitudinal study of the perimenopausal transition: altered profiles of steroid and pituitary hormones, SHBG and bone mineral density. Maturitas 21: 103-113

37. Recker R, Lappe J, Davies K et al. (2000) Characterization of perimenopausal bone loss: a prospective study. J Bone Miner Res 15: 1965-1973

38. Clements D, Compston JE, Evans C et al. (1993) Bone loss in normal British women: a 5-year follow-up. Br J Radiol 66: $1134-1137$

39. Pouilles JM, Tremollieres F, Ribot C (1994) Effect of menopause on vertebral bone mass: a longitudinal study. Presse Med 23: $1069-1073$

40. Sowers MF, Crutchfield M, Shapiro B et al. (1998) Urinary ovarian and gonadotrophin hormone levels in premenopausal women with low bone mass. J Bone Miner Res 13: 1191-1202 